\title{
Autismo no Brasil: uma revisão sobre estudos em neurogenética
}

\section{Autism in Brazil: a review of studies in neurogenetics}

\author{
El autismo en Brasil: una revisión de \\ los estudios en neurogenética
}

\author{
Victor Gustavo Oliveira Evangelhoํㅜ, Fabrício da Mota Ramalho Costa², \\ Helena Carla Castro ${ }^{3}$, Murilo Lamim Bello ${ }^{4}$, Márcia Rodrigues Amorim ${ }^{5}$
}

1.Biomédico (Genética e Biologia molecular), Doutorando no Programa de Pós-graduação em Ciências e Biotecnologia, Universidade Federal Fluminense, Niterói-RJ, Brasil. Orcid: https://orcid.org/0000-0002$\underline{0560-0115}$

2.Biólogo, Doutor em Biologia Molecular (Fiocruz). Instituto Brasileiro de Medicina de Reabilitação Laureate International Universities, Rio de Janeiro-RJ, Brasil. Orcid: https://orcid.org/0000-0002-67576587

3.Farmacêutica, Doutora em Química Biológica (UFRJ). Laboratório de Antibióticos, Bioquímica, Ensino e Modelagem Molecular, Instituto de Biologia, Universidade Federal Fluminense, Niterói-RJ, Brasil. Orcid: https://orcid.org/0000-0001-5283-1541

4.Farmacêutico, Doutor em Ciências Farmacêuticas (UFRJ). Laboratório de Planejamento Farmacêutico e Simulação Computacional, Departamento de Fármacos e Medicamentos, Universidade Federal do Rio de Janeiro, Rio de Janeiro-RJ, Brasil. Orcid: https://orcid.org/0000-0003-0860-870X

5.Bióloga, Doutora em Ciências Biológicas (Genética; UFRJ). Laboratório de Genética Humana, Instituto de Biologia. Pós-Graduação em Neurologia/Neurociências, Universidade Federal Fluminense, Niterói-RJ, Brasil. Orcid: https://orcid.org/0000-0002-0528-0377

\begin{abstract}
Resumo
Objetivo. Identificar artigos, dissertações e teses brasileiras relacionadas a neurogenética do autismo, entre os anos de 2017 e 2020. Método. A triagem dos trabalhos publicados nas grandes áreas das ciências biológicas e ciências da saúde foi realizada por meio das bases de dados PUBMED, SCIELO e plataforma CAPES. Resultados. Foram identificados 225 trabalhos, divididos em 123 artigos científicos, indexados nas bases de dados e 102 trabalhos de pósgraduação. Os critérios de inclusão e exclusão resultaram em 14 pesquisas acerca dos fatores genéticos e neurobiológicos na população brasileira. Conclusões. $O$ crescimento exponencial de casos de autismo corrobora a relevância das pesquisas voltadas para a identificação e compreensão dos fatores moleculares no autismo. Devido à complexidade da etiologia, é fundamental a realização de mais estudos em neurogenética na população brasileira, capazes de auxiliar no desenvolvimento de testes moleculares mais efetivos no diagnóstico e tratamento deste transtorno.
\end{abstract}

Unitermos. Autismo; Brasil; Neurogenética; Ciências biológicas; Ciências da saúde

\begin{abstract}
Objective. To identify Brazilian articles, dissertations and theses related to neurogenetics of autism, between 2017 and 2020. Method. The screening of the papers published in the broad areas of biological sciences and health sciences was performed through the databases PUBMED, SCIELO and CAPES platform. Results. 225 papers were identified, divided into 123 scientific articles, indexed in the databases and 102 graduate papers. The inclusion and exclusion criteria resulted in 14 studies about genetic and neurobiological factors in the Brazilian population. Conclusions. The exponential growth of autism cases corroborates the relevance of research aimed at identifying and understanding the molecular factors in autism. Due to the complexity of etiology, it is essential to conduct further studies in neurogenetics in the Brazilian population, capable of assisting in the development of molecular tests more effective in the diagnosis and treatment of this disorder.
\end{abstract}

Keywords. Autism; Brazil; Neurogenetics; Biological Sciences; Health Sciences 


\section{Resumen}

Objetivo. Identificar artículos, disertaciones y tesis brasileñas relacionadas con la neurogenética del autismo, entre los años 2017 y 2020 . Método. El cribado de los artículos publicados en las amplias áreas de las ciencias biológicas y de la salud se realizó a través de las bases de datos PUBMED, SCIELO y la plataforma CAPES. Resultados. Se identificaron 225 trabajos, divididos en 123 artículos científicos, indexados en las bases de datos y 102 trabajos de postgrado. Los criterios de inclusión y exclusión dieron como resultado 14 estudios sobre factores genéticos y neurobiológicos en la población brasileña. Conclusiones. El crecimiento exponencial de los casos de autismo corrobora la relevancia de la investigación dirigida a identificar y comprender los factores moleculares del autismo. Debido a la complejidad de la etiología, es esencial realizar más estudios en neurogenética en la población brasileña, capaces de ayudar en el desarrollo de pruebas moleculares más eficaces en el diagnóstico y tratamiento de este trastorno.

Palabras clave. Autismo; Brasil; Neurogenetica; Ciencias Biológicas; Ciencias de la Salud

Trabalho realizado na Universidade Federal Fluminense, Niterói-RJ, Brasil.

\section{INTRODUÇÃO}

O autismo é um transtorno do desenvolvimento, caracterizado por um déficit significativo na socialização e na comunicação. Os indivíduos com o transtorno do espectro autista (TEA) podem apresentar interesses restritos, incapacidade para compreender abstrações, comportamento estereotipado ou repetitivo e alterações no processamento sensorial $^{1}$. De acordo com o Centers for Disease Control and Prevention (CDC), o autismo afeta 1 a cada 54 nascimentos, nos Estados Unidos, sendo quatro vezes mais frequente no sexo masculino ${ }^{2}$.

Segundo um estudo epidemiológico que analisou dados no período de 1990 até 2016, foi estimado que 62,1 milhões de pessoas no mundo têm o transtorno do espectro autista ${ }^{3}$. No Brasil, não há uma análise no sistema público de saúde que tenha avaliado a incidência em todo o território nacional. Contudo, estima-se que existam, aproximadamente, 2,7 
milhões de indivíduos com autismo (1\% da população), segundo dados da Organização das Nações Unidas (ONU, 2017) e dados da Global Burden of Disease Collaborative Network ${ }^{3}$.

O termo autismo significa "si mesmo" e foi criado pelo psiquiatra suíço Eugen Bleuler, em 1908, como uma condição subjacente a esquizofrenia, caracterizada pelo isolamento social. Historicamente, o autismo como conhecemos hoje, foi identificado pela também psiquiatra russa Grunya Sukhareva, em 1924, duas décadas antes de Leo Kanner, em 1943 e Hans Asperger, em $1944^{4}$.

O diagnóstico do autismo pode ser realizado antes dos três anos de idade, sendo baseado em uma análise clínicacomportamental, seguindo critérios delimitados pelo Manual de Diagnóstico e Estatístico de Transtornos Mentais (DMS) ou pela Classificação Internacional de Doenças (CID) ${ }^{5}$.

Partindo dessa abordagem, algumas políticas públicas, embora tardias, foram instituídas para garantir direitos básicos para esses indivíduos, uma delas foi a Política Nacional de Proteção dos Direitos da Pessoa com Transtornos do Espectro do Autismo 6 . E a Lei no 12.764, que para fins legais e de acesso aos atendimentos da saúde pública, através do SUS, equipararam os indivíduos com autismo à população com deficiência, permitindo usufruir das mesmas prerrogativas. Dois grandes avanços destacam-se no inciso III, que garante o acesso ao diagnóstico precoce e ao atendimento multiprofissional, bem como o inciso IV, que viabiliza o acesso educacional, profissional, a inserção no 
mercado de trabalho, o direito a previdência e assistência social.

Segundo Diretrizes de Atenção à Reabilitação da Pessoa com Transtornos do Espectro do Autismo7, pelo Ministério da Saúde, alguns indicadores devem ser analisados na atenção básica, para verificar se a criança apresenta um desenvolvimento atípico enquadrado no espectro. Essa iniciativa emerge como uma estratégia para o diagnóstico precoce, porém, ainda se trata de um desafio no país. Contudo, é comum o diagnóstico ocorrer em fases mais tardias, pois depende critérios comportamentais, havendo dificuldade profissional, devido à grande variabilidade fenotípica ${ }^{8}$.

A etiologia do autismo é complexa, influenciada por um mecanismo multifatorial, que envolve fatores genéticos e ambientais $^{9,10}$. Em cerca de $49 \%$ dos casos, o autismo é ocasionado por variantes genéticas comuns, presentes na população, mas que sozinhas, conferem um baixo risco para o desenvolvimento do transtorno, porém, possuem um fator aditivo e conferem maior risco quando o indivíduo é portador de múltiplas variantes comuns ${ }^{11}$.

Em $10 \%$ dos casos pode-se identificar mutações raras, geralmente de novo, e variações nos números de cópias CNV's (copy number variations) ${ }^{11,12}$. Alterações cromossômicas são bem descritas no autismo; incluindo algumas síndromes de microdeleção/microduplicação. Cerca de 5 a $10 \%$ dos indivíduos com transtornos do espectro autista apresentam síndromes/distúrbios monogênicos ${ }^{12}$. 
Atualmente, existem três grandes biobancos que indicam quais genes estão mais relacionados com o autismo. Dentre eles, o repositório Sfari Gene possui mais de mil genes com potenciais implicações no autismo, que atribui pontuações de 1 a 6 , indicando a força da evidência dos genes reportado por estudos científicos e a relação genetranstorno ${ }^{13}$.

Outra iniciativa importante é a Simons Foundation Powering Autism Research for Knowledge (SPARK), uma organização de pesquisa que divulga anualmente os genes e variações do número de cópias mais relacionados com 0 autismo, com base em testes genéticos de voluntários e familiares de indivíduos com o transtorno (Figura 1).

Figura 1. Representação criada com a ferramenta Biorender, baseada nos dados da lista divulgada pelo projeto SPARK, contendo 156 genes, e variações do número de cópias, relacionados ao neurodesenvolvimento do autismo (Adaptado de SPARK, $\left.2020^{14}\right)$.

VARIAÇÃO DO NÚMERO DE CÓPIAS (CNV'S)

$1 \mathrm{q} 21.1,2 \mathrm{p} 16.3,3 \mathrm{q} 29,5 \mathrm{q} 35,7 \mathrm{q} 11.23,8 \mathrm{p} 23.1,15 \mathrm{q} 11.2$ BP1-BP2 (deleção), 15q11.2-q13.1, 15q13.3, 15q15, $16 \mathrm{p} 11.2,16 \mathrm{p} 12.1,16 \mathrm{p} 13.3,17 \mathrm{p} 11.2,17 \mathrm{q} 11.2,17 \mathrm{q} 12$ $17 \mathrm{q} 21.3,22 \mathrm{q} 11.2$ e $22 \mathrm{q} 13.3$.

\section{GENES}

ACTB, ADNP, ADSL, AFF2, AHDC1, ALDH5A1, ANK2, ANK3, ANKRD11, ARHGEF9, ARID1B, ARX, ASH1L, ASXL3, ATRX, AUTS2, BAZ2B, BCKDK, BCL11A, BRAF, BRSK2, CACNA1C CASK CDKL5, CHAMP1, CHD2, CHD3, CHD7 CHD8, CIC CNOT3, CREBBP, CTNNB1, CTCF, CUL3, DDX3X, DHCR7, DLG4, DMPK, DNMT3A, DSCAM, DYRK1A, EBF3, EHMT1, EP300, FMR1, FOXG1, FOXP1, GIGYF1, GIGYF2, GRIN2B, HECTD4, HIVEP2, HNRNPH2, HNRNPU, HRAS, IRF2BPL, IQSEC2, KANSL1, KCNB1, KDM3B, KDM6B, KIAA2022, KRAS, KMT2A, KMT2C, LZTR1, MAGEL2, MAP2K1, MAP2K2, MBD5, MBOAT7, MECP2, MED13, MED13L, MEIS2, MYT1L, NAA15, NBEA, NCKAP1, NF1, NIPBL, NLGN2, NLGN3, NRAS, NR4A2, NRXN1, NRXN2, NRXN3, NSD1, PACS1, PCDH19, PHF21A PHF3, PHIP POGZ POMGNT1, PPP1CB PPP2R5D, PSMD12 PTCHD1, PTPN11, PTEN, RAF1, RAI1, REIN, RERE, RFX3, RIMS1, RIT1, RORB SCN1A SCN2A SCN8A SETBP1, SETD2, SETD5, RIMS1, RIT1, RORB, SCN1A, SCN2A, SCN8A, SETBP1, SETD2, SETD5,
SHANK2, SHANK3, SHOC2, SIN3A, SLC6A1, SLC9A6 (NHE6), SMARCC2, SON, SOS1, SOS2, SOX5, SPAST, SRCAP, STXBP1, KMT5B (SUV420H1), SYNGAP1, TAOK1, TANC2, TBCK, TBR1, TCF20, TCF4, TLK2, TRIO, TRIP12, TSC1, TSC2, TSHZ3, UBE3A, UPF3B, VPS13B, WAC, WDFY3, ZBTB20, ZNF292 e ZNF462. 
Nesse contexto multifatorial, os riscos ambientais são menos compreendidos, mas algumas pesquisas indicam que a utilização de anticonvulsivantes e estabilizador de humor, como o ácido valproico na gestação, dentre outros agentes químicos, podem, em casos raros, afetar o desenvolvimento intrauterino e predispor ao autismo ${ }^{15-17}$.

Dessa forma, os objetivos desse estudo foram: identificar os artigos, dissertações e teses brasileiras relacionadas a neurogenética do autismo, publicados no período de 2017 a 2020, relacionar as publicações e analisar as perspectivas das pesquisas científicas sobre o autismo no país.

\section{MÉTODO}

Por meio das bases de dados Scielo, Pubmed e da plataforma CAPES, realizou-se o levantamento de artigos, dissertações e teses relacionadas ao autismo.

Nessas bases de dados utilizou-se os descritores: "genética" e "autismo"; "prevalência" e "autismo"; "autismo"; "Transtorno do espectro autista" e "TEA"; associados a "Brasil". Esses descritores foram utilizados em inglês e português, limitando-se aos termos identificados em títulos, resumos e assuntos, quando necessário. Posteriormente, os artigos foram analisados na ferramenta Rayyan, para automatizar a escolha dos trabalhos ${ }^{18}$. 


\section{Critérios de Inclusão}

Os critérios para a inclusão dos artigos, dissertações e teses foram restritos a produção científica no Brasil, no período de 2017 e 2020. Foram selecionados trabalhos da grande área de ciências biológicas e ciências da saúde em humanos ou modelos celulares derivados.

\section{Critérios de Exclusão}

Os trabalhos excluídos foram baseados na duplicidade do trabalho em diferentes bases de dados e utilização de modelo animal para o estudo. Foram descartadas também as revisões bibliográficas.

\section{Roteiro do Processo de Seleção}

Inicialmente, a seleção dos trabalhos acadêmicos resultou em 225 trabalhos identificados, divididos em 123 artigos científicos, indexados nas bases de dados PUBMED e SCIELO e 102 trabalhos de pós-graduação (teses e dissertações), depositadas na plataforma da CAPES.

Todos os artigos foram salvos no formato Bibtex e, posteriormente direcionados para a plataforma Rayyan, onde realizou-se a mineração de texto, por meio dos critérios estabelecidos. Dos 127 artigos identificados no levantamento, 74 foram excluídos por não estarem dentro dos critérios de inclusão (Figura 2). Posteriormente, dos 53 artigos selecionados com estudos em humanos, pela análise do título e resumo, apenas 12 foram elegíveis para este trabalho e incluídos na revisão. 
A seleção de trabalhos de pós-graduação foi feita manualmente na plataforma da CAPES. Dentre as dissertações e teses selecionadas, 102 trabalhos de pósgraduação foram selecionados. A partir da primeira análise, 28 trabalhos foram selecionados para análise do título e do resumo. Nesse processo, trabalhos que utilizavam modelo animal, ou já publicados em formato de artigo, ou que não estavam disponíveis no site da capes, foram descartados. Por fim, 2 dissertações foram selecionadas para a inclusão na revisão.

Figura 2. Fluxograma geral das etapas realizadas na seleção dos artigos.

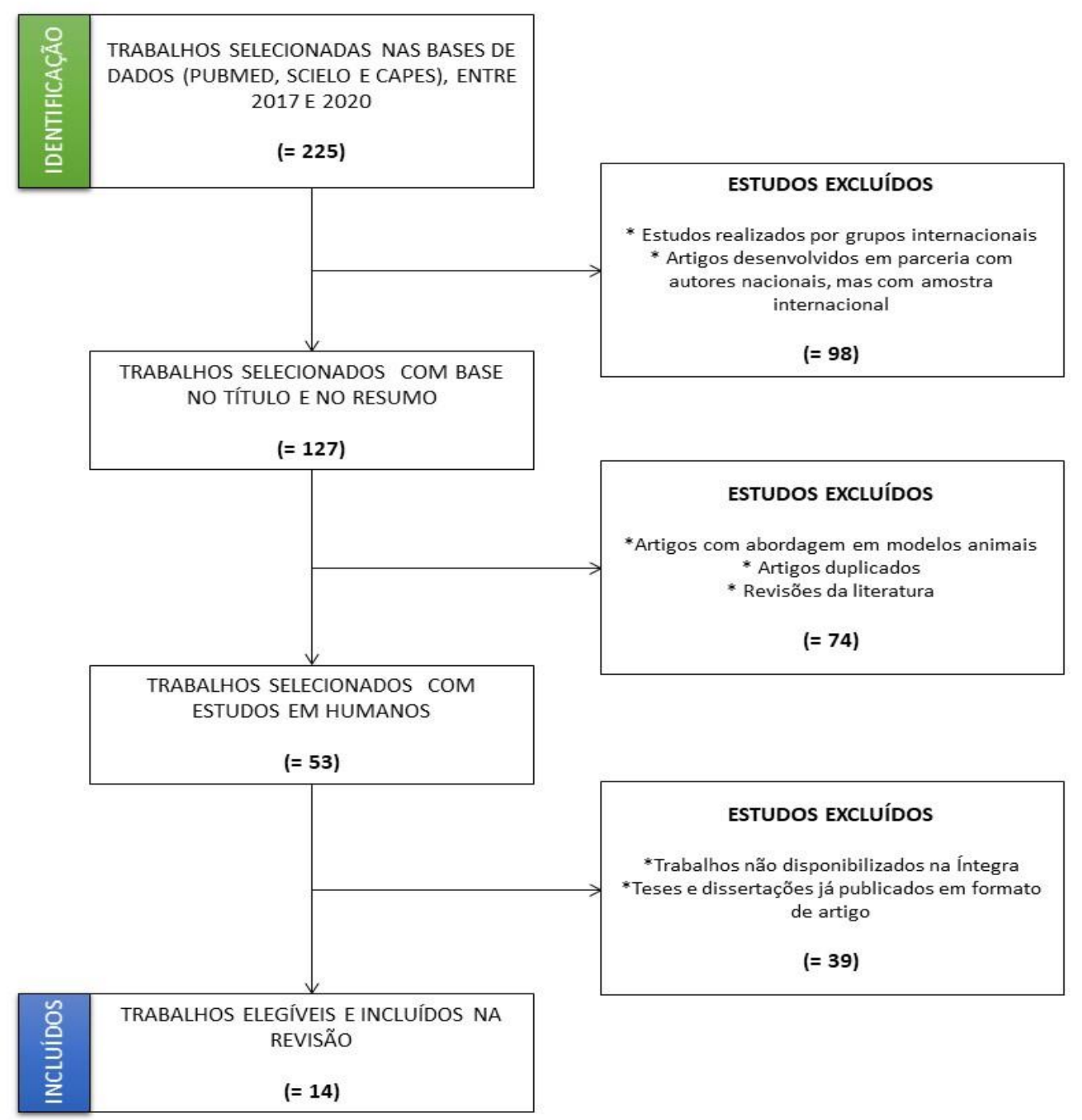


Ao final da aplicação dos critérios de seleção, 14 trabalhos de pesquisa no Brasil foram elegíveis. Os dados foram subdivididos em seções, constando os autores, título do estudo, objetivo da pesquisa e os principais resultados obtidos na pesquisa (Quadro 1).

Quadro 1. Dados extraídos a partir dos trabalhos de pesquisas em autismo no Brasil.

\begin{tabular}{|c|c|c|c|}
\hline AUTORES & TIPO & TÍTULO ORIGINAL & RESULTADOS / CONCLUSÃO \\
\hline Guloksuz et al. ${ }^{19}$ & Artigo & $\begin{array}{l}\text { Elevated plasma concentrations } \\
\text { of S100 calcium-binding protein } \\
\mathrm{B} \text { and tumor necrosis factor } \\
\text { alpha in children with autism } \\
\text { spectrum disorders }\end{array}$ & $\begin{array}{l}\text { As concentrações de } \mathrm{S} 100 \mathrm{~B} \text { foram maiores em } \\
\text { crianças com autismo grave em comparação com } \\
\text { crianças com autismo leve-moderado. Indicando } \\
\text { a relação da proteína codificada a partir desse } \\
\text { gene no transtorno do espectro autista. }\end{array}$ \\
\hline $\begin{array}{l}\text { Sánchez-Sánchez } \\
\text { et al. }{ }^{20}\end{array}$ & Artigo & $\begin{array}{lrr}\text { Rare RELN Variants } & \text { Affect } \\
\text { Reelin-DAB1 } & \text { Signal } \\
\text { Transduction in } & \text { Autism } \\
\text { Spectrum Disorder } & \\
& & \\
& \end{array}$ & $\begin{array}{l}\text { As variantes identificadas são deletérias e levam } \\
\text { à secreção diminuída da Relina e à transdução do } \\
\text { sinal Reelin-DAB1 prejudicada. Além disso, os } \\
\text { resultados sugerem que a superativação da via } \\
\text { mTORC1 pode funcionar como um segundo } \\
\text { evento de impacto contribuindo para a regulação } \\
\text { negativa da cascata Reelin-DAB1 em NPCs } \\
\text { derivados do paciente. }\end{array}$ \\
\hline Ribeiro et al. ${ }^{21}$ & Artigo & $\begin{array}{l}\text { Evidence for Association } \\
\text { Between OXTR Gene And ASD } \\
\text { Clinical Phenotypes }\end{array}$ & $\begin{array}{l}\text { As descobertas gerais sugerem que o gene OXTR } \\
\text { pode desempenhar um papel no diagnóstico de } \\
\text { autismo e em alguns de seus fenótipos clínicos, } \\
\text { apoiados por estudos anteriores. Além disso, na } \\
\text { análise de base familiar, foi observada uma } \\
\text { tendência à associação com a susceptibilidade ao } \\
\text { TEA para o rs1042778 (alelo } G \text { ) (P=0,066). Em } \\
\text { uma abordagem bioinformática, demonstrou-se } \\
\text { que o alelo rs1042778 (G) é determinante para a } \\
\text { ligação do fator de transcrição MAZ. }\end{array}$ \\
\hline Guzman et al. ${ }^{22}$ & Artigo & $\begin{array}{l}\text { Identification of Alterations } \\
\text { Associated with Age in The } \\
\text { Clustering Structure of } \\
\text { Functional Brain Networks }\end{array}$ & $\begin{array}{l}\text { Identificou-se duas regiões cerebrais para as } \\
\text { quais os agrupamentos mudam com o tempo, } \\
\text { sendo elas, o giro temporal médio esquerdo e o } \\
\text { putâmen esquerdo. Como o índice de adequação } \\
\text { da rede está associado à integração e à } \\
\text { segregação. O resultado sugere que a região } \\
\text { cerebral identificada desempenha um papel no } \\
\text { desenvolvimento de sistemas cerebrais. }\end{array}$ \\
\hline Russo et al. ${ }^{23}$ & Artigo & $\begin{array}{l}\text { Modeling the Interplay Between } \\
\text { Neurons and Astrocytes in } \\
\text { Autism Using Human Induced } \\
\text { Pluripotent Stem Cells }\end{array}$ & $\begin{array}{l}\text { Os neurônios derivados do TEA tiveram uma } \\
\text { diminuição significativa na expressão gênica } \\
\text { sináptica e nos níveis de proteínas, liberação de } \\
\text { neurotransmissores de glutamato e, } \\
\text { consequentemente, redução na taxa de disparo } \\
\text { espontâneo. Observou-se que os astrócitos } \\
\text { derivados do autismo interferiam no } \\
\text { desenvolvimento neuronal adequado. Em } \\
\text { contraponto, os astrócitos derivados do controle } \\
\text { resgataram o fenótipo neuronal morfológico e os } \\
\text { defeitos da sinaptogênese. } \\
\end{array}$ \\
\hline $\begin{array}{l}\text { Griesi-Oliveira et } \\
\text { al. }{ }^{24}\end{array}$ & Artigo & $\begin{array}{l}\text { Actin Cytoskeleton Dynamics in } \\
\text { Stem Cells from Autistic } \\
\text { Individuals }\end{array}$ & $\begin{array}{l}\text { Foi observado que as células-tronco de sete } \\
\text { indivíduos com TEA apresentaram alterações } \\
\text { dinâmicas da reconstrução de filamentos de } \\
\text { actina. Os resultados sugerem que, pelo menos } \\
\text { para um subgrupo de pacientes com TEA, a } \\
\text { dinâmica da polimerização da actina é } \\
\text { prejudicada. }\end{array}$ \\
\hline
\end{tabular}


Quadro 1 (cont). Dados extraídos a partir dos trabalhos de pesquisas em autismo no Brasil.

\begin{tabular}{|c|c|c|c|}
\hline AUTORES & TIPO & TÍTULO ORIGINAL & RESULTADOS / CONCLUSÃO \\
\hline Antunes et al. ${ }^{25}$ & Artigo & $\begin{array}{lr}\text { Mirror Neurons } & \text { Modeled } \\
\text { Through } & \text { Spike-Timing- } \\
\text { Dependent Plasticity Are } \\
\text { Affected by Channelopathies } \\
\text { Associated with Autism } \\
\text { Spectrum Disorder. }\end{array}$ & $\begin{array}{l}\text { Os resultados de simulação mostraram que } \\
\text { correntes catiônicas ativadas por hiperpolarização } \\
\text { prejudicada afetavam a função dos neurônios } \\
\text { espelho entre os pares de neurônios neocorticais } \\
\text { acoplados pela plasticidade sináptica dependente } \\
\text { do tempo (STDP) em indivíduos autistas. }\end{array}$ \\
\hline Ramos et al. ${ }^{26}$ & Artigo & $\begin{array}{l}\text { Abnormal Cortico-Cerebellar } \\
\text { Functional Connectivity in } \\
\text { Autism Spectrum Disorder }\end{array}$ & $\begin{array}{l}\text { Descobriu-se que pessoas com autismo tiveram } \\
\text { conectividade funcional diminuída entre o } \\
\text { cerebelo e regiões corticais envolvidas em } \\
\text { sistemas cognitivos que contribuem para as } \\
\text { funções comumente afetadas no autismo. }\end{array}$ \\
\hline Ferreira et al. ${ }^{27}$ & Artigo & $\begin{array}{l}\text { Screening for FMR1 Expanded } \\
\text { Alleles in Patients with Autism } \\
\text { Spectrum Disorders in } \\
\text { Manaus, Northern Brazil }\end{array}$ & $\begin{array}{l}\text { A frequência de expansões de CGG foram } \\
\text { verificadas em } 90 \text { homens com autismo por } \\
\text { análise molecular. Quatro deles tinham alelos } \\
\text { intermediários e outros quatro apresentaram } \\
\text { alelos pré-estratificados. Não foram encontrados } \\
\text { alelos de mutação completa. }\end{array}$ \\
\hline Sampaio et al. ${ }^{28}$ & Artigo & $\begin{array}{l}\text { Generation of An Induced } \\
\text { Pluripotent Stem Cell Line } \\
\text { from A Patient with Autism } \\
\text { Spectrum Disorder and } \\
\text { SCN2A Haploinsufficiency }\end{array}$ & $\begin{array}{l}\text { A linha iPSC gerada expressa marcadores de } \\
\text { pluripotência, apresenta um cariótipo normal e foi } \\
\text { capaz de se diferenciar nas três camadas } \\
\text { germinativas. Esta linha iPSC é uma ferramenta } \\
\text { útil para modelar estudos de rastreamento de } \\
\text { drogas e autismo. }\end{array}$ \\
\hline Schuch et al. ${ }^{29}$ & Artigo & $\begin{array}{l}\text { Analysis of A Protein Network } \\
\text { Related to Copy Number } \\
\text { Variations In } \\
\text { Spectrum Disorder }\end{array}$ & $\begin{array}{l}\text { Entre as } 40 \text { crianças analisadas, encontramos } 14 \\
\text { CNVs potencialmente patogênicas, incluindo } \\
\text { aquelas previamente associadas ao autismo } \\
\text { (localizadas nas regióes } 16 \text { p } 11.2,15 q 11.2 \text { e } \\
\text { 7p21). A rede de autismo relacionada à CNV } \\
\text { compreendia } 90 \text { proteínas e } 754 \text { nós e indicou a } \\
\text { família de receptores olfativos como uma via } \\
\text { significativa no TEA. }\end{array}$ \\
\hline Chaves et al. ${ }^{30}$ & Artigo & $\begin{array}{l}\text { Long Contiguous Stretches of } \\
\text { Homozygosity Detected by } \\
\text { Chromosomal Microarrays } \\
\text { (CMA) in Patients with } \\
\text { Neurodevelopmental } \\
\text { Disorders in The South of } \\
\text { Brazil }\end{array}$ & $\begin{array}{l}\text { Em 95\% dos pacientes, pelo menos um longo } \\
\text { trecho contíguo de homozigose (LCSH) foi } \\
\text { detectado, um total de } 1478 \text { LCSH em } 407 \\
\text { pacientes. Em 2,6\%, os achados foram } \\
\text { sugestivos de UPD. Por cerca de } 8,5 \% \text {, o LCSH } \\
\text { sugere filhos do primeiro ao quinto ano, com } \\
\text { maior probabilidade de ter um impacto clínico. }\end{array}$ \\
\hline Nascimento ${ }^{31}$ & Dissertação & $\begin{array}{lrr}\text { Análise } & \text { Cromossômica } & \text { Por } \\
\text { Microarranjos } & & \text { Na } \\
\text { Investigação } & \text { De } & \text { Alterações } \\
\text { Genômicas } & \text { Estruturais } & \text { No } \\
\text { Transtorno } & \text { Do } & \text { Espectro } \\
\text { Autista - TEA } & & \end{array}$ & $\begin{array}{l}\text { Observou-se predomínio de CNVs envolvendo os } \\
\text { cromossomos } \mathrm{X}, 14,15 \text { e } 22 \text {. Dentre todas as } \\
\text { CNVs observadas neste estudo observamos } \\
\text { predomínio de CNVs de ganhos em relação às } \\
\text { CNVs de perdas e os genes que se destacaram } \\
\text { foram: ST6GAL2, NUP155, WDR70, CHRNA7, } \\
\text { TPPP, ZNF630, SSX6 e SPACA5. }\end{array}$ \\
\hline Gonçalves 32 & Dissertação & $\begin{array}{llr}\text { Pesquisa } & \text { De } & \text { Alterações } \\
\text { Genéticas Em Pacientes Com } \\
\text { Transtorno Do } & \text { Espectro } \\
\text { Autista (TEA) } & \text { Em Uma } \\
\text { Amostra de Minas Gerais, } \\
\text { Brasil: Uma } & \text { Uvaliação } \\
\text { Genético-Molecular } & \end{array}$ & $\begin{array}{l}\text { Não foram detectadas alterações numéricas e } \\
\text { estruturais ao cariótipol. Um }(1,5 \%) \text { de } 65 \\
\text { pacientes apresentou Síndrome do X frágil. Dois } \\
\text { pacientes tiveram diagnóstico molecular } \\
\text { estabelecido com o kit SALSA MLPA P245-B1, } \\
\text { uma deleção em 15q24, presente em uma } \\
\text { paciente do sexo masculino, e uma outra deleção } \\
\text { em 22q13, presente em um paciente do sexo } \\
\text { feminino. }\end{array}$ \\
\hline
\end{tabular}

* CNVs: variação do número de cópias; iPSCs: Células tronco pluripotente induzidas; LCSH: Longos Trechos Contínuos De Homozigose; MLPA: Amplificação de Múltiplas Sondas Dependente de Ligação; TDAH: transtorno do déficit de atenção e hiperatividade; TEA: Transtorno do espectro autista; UPD: Dissomia uniparental. 
Os estudos foram subdivididos por área, sendo possível verificar a maior e menor subárea de pesquisa, dentro do tempo definido para levantamento (Gráfico 1). A partir dos 14 trabalhos selecionados, foi possível delimitar 0 enquadramento da área de estudo especificada por meio da metodologia utilizada.

Gráfico 1. Percentual de artigos selecionados por tipo de análise.

Análise Molecular

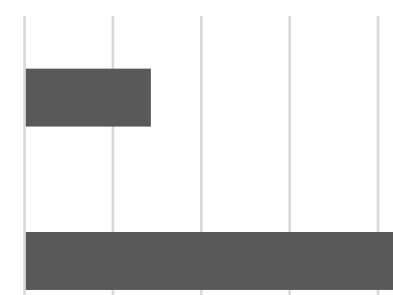

Neurobiologia

Análise genética / Células Tronco Pluripotentes

Análise genética

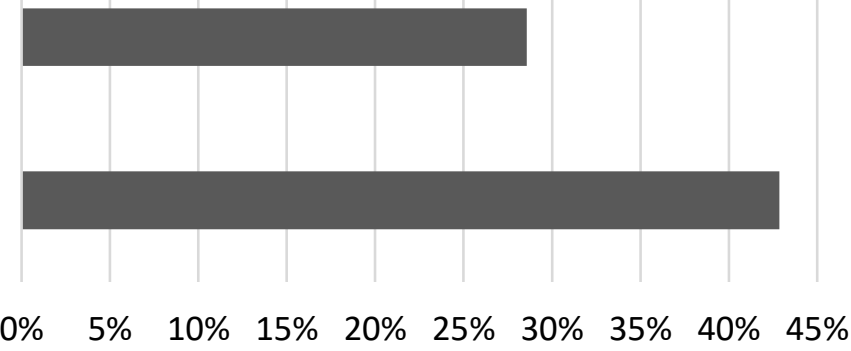

\section{DISCUSSÃO}

As pesquisas elencadas na revisão analisaram a neurofisiologia do autismo por meio de variadas interfaces, dentre elas, as análises celulares, genéticas e neurofisiológicas. Nesse contexto, cabe ressaltar a variabilidade da etiologia genética que está subjacente ao transtorno em indivíduos não relacionados. As análises genéticas e a manipulação de células humanas realizadas no Brasil indicaram variações no número de cópias (CNVs) e 
dezenas de genes relacionados a predisposição para o desenvolvimento de autismo.

Alguns genes estudados corroboram com os achados presentes na literatura internacional, ao identificarem alteração da expressão do gene Reelina ( $R E L N$ ), via RhoA e via mTOR ${ }^{20,24}$. A Reelina é fundamental para a laminação do sistema nervoso central (SNC), a via mTOR está implicada na poda neural e no neurodesenvolvimento e a via RhoA é fundamental para que ocorra a migração celular e a motilidade do citoesqueleto no SNC ${ }^{33-35}$.

Conforme identificando no estudo do gene OXTR, que codifica o receptor de ocitocina, os resultados gerais indicam alguns polimorfismos implicados na predisposição ao autismo, no fenótipo observado, na interação social e outros processos neurobiológicos $21,29,31$.

Contudo, estudos moleculares voltados para a identificação da sobreposição genética entre o autismo e outros transtornos e síndromes são limitados no Brasil. Internacionalmente, algumas síndromes frequentemente observadas no autismo sindrômico são bem estudadas, como a síndrome de Rett e síndrome do X-frágil. No Brasil, os testes genéticos para essas duas síndromes são comumente solicitados na prática clínica, quando além de outros aspectos clínicos, há suspeita do transtorno em meninas e meninos, respectivamente ${ }^{24}$.

O autismo pode estar presente em outras condições monogênicas ou alterações cromossômicas, como: Síndrome de Cohen, Síndrome de Cornelia de Lange, Esclerose 
Tuberosa, Síndrome de Angelman, Síndrome De CHARGE, Neurofibromatose Tipo 1, Síndrome de Down, Síndrome de Noonan, Síndrome de Williams, Síndrome de Deleção 22q11.2, Síndrome de Joubert, Phelan-McDermid e outras ${ }^{36}$, sendo relevante o estudo mais amplo do autismo sindrômico e seus mecanismos subjacentes.

A heterogeneidade desta neuroatipicidade é influenciada por múltiplos genes. Mutações raras, algumas ainda não identificadas na literatura, são um grande desafio no diagnóstico clínico, muitas vezes inviabilizando dissociar os indicadores primários dessas síndromes, das características do autismo que aparecem de forma secundária.

$\mathrm{Na}$ complementaridade das pesquisas genéticas, novas plataformas de mimetização de transtornos surgiram nos últimos tempos. Essa metodologia é realizada por meio da reprogramação de células somáticas, provenientes de indivíduos com a condição desejada para estudo e auxiliam nas análises que necessitariam de estudos invasivos ao longo do desenvolvimento embrionário 24,28 .

A utilização de células somáticas é importante para a compreensão da diferenciação e migração de progenitores neuronais, por meio da indução dessas plataformas celulares em progenitores neurais ${ }^{23}$.

Os estudos que abordaram a neuromorfofisiologia indicaram alterações na conectividade entre o cerebelo e as regiões corticais em indivíduos com autismo, bem como, alterações nos neurônios espelho $22,25,26$. Contudo, diversas 
regiões estão relacionadas ao comportamento autista, o que torna o mapeamento dessas modificações morfológicas um desafio para o diagnóstico ${ }^{37}$.

Além desses achados, as alterações em neurotransmissores são descritas em estudos internacionais e se sobrepõe em outros transtornos. Como por exemplo, a sinapse GABAérgica, importante neurotransmissor inibitório, influindo na sinalização do ácido gamma-aminobutírico (GABA) e relacionando-se com a sinalização em diferentes partes do cérebro. Os receptores GABA parecem ter um papel central na ansiedade e perturbações nestes alvos moleculares podem originar epilepsia, comumente presente em indivíduos com $\operatorname{TEA}^{38}$. Dados recentes reforçam as alterações na sinalização GABAérgica e glutamatérgica em minicérebros derivados de indivíduos com autismo, o que contribui para as alterações fenótipicas observadas nesses indivíduos ${ }^{39}$.

As alterações neurofisiológicas estão intrinsicamente relacionadas a alterações psiquiátricas para além do autismo. Embora pouco explorado nas pesquisas brasileiras, as alterações genéticas identificadas no TEA também estão sobrepostas a outras condições neuropsiquiátricas associadas, como: esquizofrenia, o transtorno bipolar, TDAH, Epilepsia, Transtorno de ansiedade, Transtorno depressivo e outros $^{40}$. Acredita-se que até $88 \%$ dos autistas apresentem alterações no processamento sensorial ${ }^{41}$. Cerca de $27 \%$ dos autistas possuem transtorno bipolar $^{42}$ e até $35 \%$ desses indivíduos sofrem com esquizofrenia43,44. A epilepsia, 
condição comumente relacionada ao autismo, afeta até 46\% desses indivíduos. Além disso, cerca de $10 \%$ das pessoas identificadas com autismo entre 40 e 60 anos desenvolvem Alzheimer ${ }^{45}$.

Mesmo com todas as limitações no fomento da pesquisa no país, há pesquisas brasileiras nas áreas da saúde e ciências biológicas com resultados relevantes e que corroboram dados internacionais. Com relação aos artigos selecionados, poucos estudos em neurogenética humana foram selecionados, utilizando o intervalo entre os anos de 2017 e 2020. Esse fato pode ser justificado, em parte, devido aos processos e normas estabelecidas para a realização de pesquisas científicas utilizando seres humanos. Tendo em vista que os projetos experimentais ou observacionais em humanos devem ser aprovados por comitês de ética em pesquisa, sendo necessário cumprir exigências que garantam a proteção, segurança e dignidade dos participantes dessas pesquisas. Dessa forma, a pesquisa experimental em animais acaba sendo mais viável em muitos casos, mas a variação no organismo modelo pode não mimetizar as mesmas respostas observadas em humanos.

\section{CONCLUSÃO}

Embora muitos estudos estejam direcionados a compreensão das bases neurogenéticas do autismo, ainda se compreende pouco da sua etiologia. Nesse contexto, o crescimento exponencial de casos de autismo corrobora para 
a relevância mundial das pesquisas voltadas para a identificação e compreensão dos fatores moleculares.

Com base na revisão realizada, foram identificadas 14 pesquisas brasileiras acerca dos fatores genéticos e neurobiológicos do TEA. As alterações genéticas interferem em vias bioquímicas responsáveis pelo desenvolvimento atípico do sistema nervoso central nos indivíduos com o transtorno. A respeito da transversalidade dos trabalhos, os instrumentos utilizados e os resultados obtidos estavam de acordo com a literatura internacional.

Por fim, evidencia-se a importância da abordagem neurogenética humana em novos estudos na população brasileira. Tendo em vista que, novas pesquisas podem propiciar a identificação de biomarcadores capazes de auxiliar no desenvolvimento de testes mais efetivos no diagnóstico mais precoce.

\section{AGRADECIMENTOS}

Os autores agradecem à CAPES (Coordenação de Aperfeiçoamento de Pessoal de Nível Superior), CNPq (Conselho Nacional de Desenvolvimento Científico e Tecnológico), FAPERJ (Fundação de Amparo à Pesquisa do Estado do Rio de Janeiro), UFF (Universidade Federal Fluminense) e UFRJ (Universidade Federal do Rio de Janeiro), Brasil. 


\section{REFERÊNCIAS}

1.Robertson CE, Baron-Cohen S. Sensory perception in autism. Nature Rev Neurosci 2017;18:671-84. https://doi.org/10.1038/nrn.2017.112 2. Maenner MJ, Shaw KA, Baio J, Washington A, Patrick M, DiRienzo M, et al. Prevalence of Autism Spectrum Disorder Among Children Aged 8 Years - Autism and Developmental Disabilities Monitoring Network, 11 Sites, United States, 2016. MMWR Surv Summ 2020;69:1-12. https://doi.org/10.15585/mmwr.ss6904a1

3.Olusanya BO, Davis AC, Wertlieb D, Boo N-Y, Nair MKC, Halpern R, et al. Developmental disabilities among children younger than 5 years in 195 countries and territories, 1990-2016: a systematic analysis for the Global Burden of Disease Study 2016. Lancet Glob Health 2018;6:e1100-21. https://doi.org/10.1016/S2214-109X(18)30309-7

4.Posar A, Visconti P. Tribute to Grunya Efimovna Sukhareva, the woman who first described infantile autism. J Ped Neurosci 2017;12:300-1. https://doi.org/10.4103/jpn.JPN 4617

5.Grant JE, Chamberlain SR. Expanding the definition of addiction: DSM-5 vs. ICD-11. CNS Spectrums 2016;21:300-3. https://doi.org/10.1017/S1092852916000183

6.Brasil. Política Nacional de Proteção dos Direitos da Pessoa com Transtorno do Espectro Autista, e altera o $\S 30$ do art. 98 da Lei no 8.112, de 11 de dezembro de 1990. Lei n 12.764. Brasília. 2012. http://www.planalto.gov.br/ccivil 03/ ato2011-

2014/2012/lei/l12764.htm

7.Brasil. Ministério Da Saúde. Secretaria de Atenção à Saúde. Departamento de Ações Programáticas Estratégicas. Área Técnica de Saúde Da Pessoa Com Deficiência. Diretrizes de Atenção à Reabilitação Da Pessoa Com Transtornos Do Espectro Do Autismo (TEA). Brasília: MS; 2014.

https://bvsms.saude.gov.br/bvs/publicacoes/diretrizes atencao reabi litacao pessoa autismo.pdf

8.Blumberg SJ, Zablotsky B, Avila RM, Colpe LJ, Pringle BA, Kogan MD. Diagnosis lost: Differences between children who had and who currently have an autism spectrum disorder diagnosis. Autism 2016;20:783-95. https://doi.org/10.1177/1362361315607724

9.Rylaarsdam L, Guemez-Gamboa A. Genetic Causes and Modifiers of Autism Spectrum Disorder. Front Cell Neurosci 2019;13:1-15. https://doi.org/10.3389/fncel.2019.00385

10.Takahashi N, Harada T, Nishimura T, Okumura A, Choi D, Iwabuchi $\mathrm{T}$, et al. Association of Genetic Risks with Autism Spectrum Disorder and Early Neurodevelopmental Delays among Children without Intellectual Disability. JAMA Network Open 2020;3:1-9. https://doi.org/10.1001/jamanetworkopen.2019.21644

11.Gaugler T, Klei L, Sanders SJ, Bodea CA, Goldberg AP, Lee AB, et al. Most genetic risk for autism resides with common variation. Nat Gen 2014;46:881-5. https://doi.org/10.1038/ng.3039

12.Wiśniowiecka-Kowalnik B, Nowakowska BA. Genetics and epigenetics of autism spectrum disorder-current evidence in the field. 
00480-w

13.Abrahams BS, Arking DE, Campbell DB, Mefford HC, Morrow EM, Weiss LA, et al. SFARI Gene 2.0: A community-driven knowledgebase for the autism spectrum disorders (ASDs). Molecular Autism 2013;4:2-4. https://doi.org/10.1186/2040-2392-4-36

14. Feliciano P, Daniels AM, Snyder LG, Beaumont A, Camba A, Esler A, et al. SPARK: A US Cohort of 50,000 Families to Accelerate Autism Research. Neuron 2018;97:488-93.

https://doi.org/10.1016/j.neuron.2018.01.015

15.Sandin S, Lichtenstein P, Kuja-Halkola R, Larsson H, Hultman CM, Reichenberg A. The Familial Risk of Autism. JAMA 2014;311:1770. https://doi.org/10.1001/jama.2014.4144

16.Zhao $\mathrm{H}$, Wang $\mathrm{Q}$, Yan T, Zhang $\mathrm{Y}, \mathrm{Xu} \mathrm{H}, \mathrm{Yu} \mathrm{H}$, et al. Maternal valproic acid exposure leads to neurogenesis defects and autism-like behaviors in non-human primates. Translational Psychiatry [Internet]. 2019; 21:9(1). https://doi.org/10.1038/s41398-019-0608-1

17. Modabbernia A, Velthorst E, Reichenberg A. Environmental risk factors for autism: an evidence-based review of systematic reviews and meta-analyses. Mol Autism 2017;8:13.

https://doi.org/10.1186/s13229-017-0121-4

18. Ouzzani M, Hammady H, Fedorowicz Z, Elmagarmid A. Rayyan-a web and mobile app for systematic reviews. Syst Rev 2016;5:1-10. https://doi.org/10.1186/s13643-016-0384-4

19.Guloksuz SA, Abali O, Cetin EA, Gazioglu SB, Deniz G, Yildirim A, et al. Elevated plasma concentrations of $\mathrm{S} 100$ calcium-binding protein $\mathrm{B}$ and tumor necrosis factor alpha in children with autism spectrum disorders. Rev Bras Psiquiatr 2017;39:195-200.

https://doi.org/10.1590/1516-4446-2015-1843

20.Sánchez-Sánchez SM, Magdalon J, Griesi-Oliveira K, Yamamoto GL, Santacruz-Perez C, Fogo M, et al. Rare RELN variants affect ReelinDAB1 signal transduction in autism spectrum disorder. Hum Mutat 2018;39:1372-83. https://doi.org/10.1002/humu.23584

21.Ribeiro LOP, Vargas-Pinilla P, Kappel DB, Longo D, Ranzan J, Becker MM, et al. Evidence for Association Between OXTR Gene and ASD Clinical Phenotypes. J Mol Neurosci 2018;65:213-21. https://doi.org/10.1007/s12031-018-1088-0

22.Guzman GEC, Sato JR, Vidal MC, Fujita A. Identification of alterations associated with age in the clustering structure of functional brain networks. PLoS ONE 2018;13:1-14. https://doi.org/10.1371/journal.pone.0195906

23.Russo FB, Freitas BC, Pignatari GC, Fernandes IR, Sebat J, Muotri $A R$, et al. Modeling the Interplay Between Neurons and Astrocytes in Autism Using Human Induced Pluripotent Stem Cells. Biol Psychiatr 2018;83:569-78. https://doi.org/10.1016/j.biopsych.2017.09.021

24.Griesi-Oliveira K, Suzuki AM, Alves AY, Mafra ACCN, Yamamoto $\mathrm{GL}$, Ezquina $\mathrm{S}$, et al. Actin cytoskeleton dynamics in stem cells from autistic individuals. Sci Rep 2018;8:1-10. https://doi.org/10.1038/s41598-018-29309-6 
25. Antunes G, Faria Da Silva SF, Simoes De Souza FM. Mirror Neurons Modeled Through Spike-Timing-Dependent Plasticity are Affected by Channelopathies Associated with Autism Spectrum Disorder. Inter J Neural Systems 2018;28:1-15.

https://doi.org/10.1142/S0129065717500587

26.Ramos TC, Balardin JB, Sato JR, Fujita A. Abnormal corticocerebellar functional connectivity in autism spectrum disorder. Front

Syst

Neurosci

2019;12:1-7.

https://doi.org/10.3389/fnsys.2018.00074

27.Ferreira JFB, Batista JS, Fantin C. Screening for fmr1 expanded alleles in patients with autism spectrum disorders in Manaus, Northern Brazil. Anais Acad Bras Cienc 2019;91:1-6. https://doi.org/10.1590/0001-3765201920180882

28.Sampaio GLA, Martins GLS, Paredes BD, Nonaka CKV, Silva KN, Rossi EA, et al. Generation of an induced pluripotent stem cell line from a patient with autism spectrum disorder and SCN2A haploinsufficiency. Stem Cell Res 2019;39:101488. https://doi.org/10.1016/j.scr.2019.101488

29.Schuch JB, Paixão-Côrtes VR, Longo D, Roman T, Riesgo RS, Ranzan $\mathrm{J}$, et al. Analysis of a Protein Network Related to Copy Number Variations in Autism Spectrum Disorder. J Mol Neurosci 2019;69:1409. https://doi.org/10.1007/s12031-019-01343-7

30.Chaves TF, Oliveira LF, Ocampos M, Barbato IT, Luca GR, Barbato Filho $\mathrm{JH}$, et al. Long contiguous stretches of homozygosity detected by chromosomal microarrays (CMA) in patients with neurodevelopmental disorders in the South of Brazil. BMC Med Genom 2019;12:1-13. https://doi.org/10.1186/s12920-019-0496-5

31. Nascimento GR. Análise Cromossômica Por Microarranjo Na Investigação De Alterações Genômicas Estruturais No Transtorno Do Espectro Autista - TEA. Ayan 2017;8:55. http://tede2.pucgoias.edu.br:8080/handle/tede/3706

32.Gonçalves MS. Pesquisa De Alterações Genéticas Em Pacientes Com Transtorno Do Espectro Autista (Tea) Em Uma Amostra De Minas Gerais, Brasil: Uma Avaliação Genético-Molecular (Dissertação). Belo Horizonte: Universidade Federal de Minas Gerais; 2019. https://sucupira.capes.gov.br/sucupira/public/consultas/coleta/trabal hoConclusao/viewTrabalhoConclusao.jsf?popup=true\&id trabalho $=79$ 24928

33.Baek ST, Copeland B, Yun E-J, Kwon S-K, Guemez-Gamboa A, Schaffer $A E$, et al. An AKT3-FOXG1-reelin network underlies defective migration in human focal malformations of cortical development. Nat Med 2015;21:1445-54. https://doi.org/10.1038/nm.3982

34.Lieberman O, Pigulevskiy I, Post M, Sulzer D, Santini E. mTOR suppresses macroautophagy during postnatal development of the striatum. BioRxiv 2019;423:536680. https://doi.org/10.1101/536680 35.Trifonova EA, Klimenko AI, Mustafin ZS, Lashin SA, Kochetov AV. The mTOR signaling pathway activity and vitamin $d$ availability control the expression of most autism predisposition genes. International J Mol Sci 2019;20:1-14. https://doi.org/10.3390/ijms20246332 
36. Richards C, Jones C, Groves L, Moss J, Oliver C. Prevalence of autism spectrum disorder phenomenology in genetic disorders: A systematic review and meta-analysis. Lancet Psychiatr 2015;2:90916. https://doi.org/10.1016/S2215-0366(15)00376-4

37.Postema MC, van Rooij D, Anagnostou E, Arango C, Auzias $\mathrm{G}$, Behrmann $\mathrm{M}$, et al. Altered structural brain asymmetry in autism spectrum disorder in a study of 54 datasets. Nature Comm 2019;10:112. https://doi.org/10.1038/s41467-019-13005-8

38. Yang S, Guo X, Dong X, Han Y, Gao L, Su Y, et al. GABAA receptor subunit gene polymorphisms predict symptom-based and developmental deficits in Chinese Han children and adolescents with autistic spectrum disorders. Sci Rep 2017;7:1-9. https://doi.org/10.1038/s41598-017-03666-0

39.Lunden JW, Durens M, Phillips AW, Nestor MW. Cortical interneuron function in autism spectrum condition. Ped Res 2019;85:146-54. https://doi.org/10.1038/s41390-018-0214-6

40.Romero M, Aguilar JM, Del-Rey-Mejías Á, et al. Psychiatric comorbidities in autism spectrum disorder: A comparative study between DSM-IV-TR and DSM-5 diagnosis. Inter J Clin Health Psychol 2016;16:266-75. https://doi.org/10.1016/j.ijchp.2016.03.001

41.El-Ansary A, Hassan WM, Qasem $H$, Das UN. Identification of biomarkers of impaired sensory profiles among autistic patients. PLoS One 2016;11:1-19. https://doi.org/10.1371/journal.pone.0164153

42.Croen LA, Zerbo O, Qian Y, Massolo ML, Rich S, Sidney S, et al. The health status of adults on the autism spectrum. Autism 2015;19:81423. https://doi.org/10.1177/1362361315577517

43. Chisholm K, Lin A, Abu-Akel A, Wood SJ. The association between autism and schizophrenia spectrum disorders: A review of eight alternate models of co-occurrence. Neurosci Biobehav Rev 2015;55:173-83. https://doi.org/10.1016/j. neubiorev.2015.04.012 44.Grove J, Ripke S, Als TD, Mattheisen M, Walters RK, Won $\mathrm{H}$, et al. Identification of common genetic risk variants for autism spectrum disorder. Nat Gen 2019;51:431-44. https://doi.org/10.1038/s41588019-0344-8

45.Plana-Ripoll O, Pedersen CB, Holtz Y, Benros ME, Dalsgaard S, Jonge $\mathrm{P}$, et al. Exploring Comorbidity Within Mental Disorders among a Danish National Population. JAMA Psychiatr 2019;76:259-70. https://doi.org/10.1001/jamapsychiatry.2018.3658 